\title{
On the Explanatory Role of Mathematics in Empirical Science
}

\begin{abstract}
This paper examines contemporary attempts to explicate the explanatory role of mathematics in the physical sciences. Most such approaches involve developing so-called mapping accounts of the relationships between the physical world and mathematical structures. The paper argues that the use of idealizations in physical theorizing poses serious difficulties for such mapping accounts. An new approach to the applicability of mathematics is proposed.
\end{abstract}

\section{Robert W. Batterman* \\ University of Western Ontario}

July 10, 2008

\section{Introduction}

Several philosophical problems exist regarding the applicability of mathematics to the natural or empirical sciences. There are questions concerning whether and how mathematics can be used in theories to represent various aspects of the physical world. There are questions about whether and how mathematics can play predictive roles in our theories about the natural world. There are other questions as well. But one problem, it seems to me, stands out as most difficult. This is the problem of providing an account of how mathematics can play explanatory roles in empirical science. Of course, this question presupposes that mathematics does play such explanatory roles,

${ }^{*}$ I would like to thank the following people for very helpful comments and criticisms: Sorin Bangu, Mark Colyvan, Nicolas Fillion, Nicholaos Jones, Joe Mendola, James Overton, and Chris Pincock. This research was supported by a grant from the Social Sciences and Humanities Research Council of Canada. 
and there is far from universal agreement on whether that presupposition is true. I will have more to say about this below.

So one question is: "Are there genuine mathematical explanations of physical phenomena?" 1 Another, and the one that will be the central concern of this paper, is: "How can we understand mathematical explanations of physical phenomena?" After briefly discussing the first question, the bulk of this paper will concentrate on the second. In particular, I will examine a set of approaches, fairly described as "mapping accounts," that aim to provide accounts of applied mathematics, including mathematical explanation. I will argue that these approaches all fail to deal with the most difficult features of mathematical explanation. This is to account for how mathematical idealizations can have a role in physical explanations. Finally, I will suggest a way to move forward - a way that requires a completely new approach to the problems of applied mathematics.

\section{Mathematical Explanations I: Entities}

Why might one think that mathematics cannot play an explanatory role in physical theory? Crudely speaking, one important reason is that mathematical objects - numbers, e.g.- - are (if they exist at all) supposed to be abstract entities. They are supposed to exist outside of the causal spacetime nexus that constitutes the backdrop of all physical theories. If mathematical objects are abstract and acausal, then, if explanation in the natural sciences is fundamentally causal, mathematical entities cannot play an explanatory role. $^{2}$ This objection is only as strong as the idea that all explanations in physical theory are causal explanations. (And, of course, even weaker, if the mathematical entities don't exist at all!)

But, it seems to me that there are very good reasons to deny that all physical explanations are causal explanations. The main reason is that if one pays attention to explanations offered by physicists and applied mathematicians it is very often the case that one finds no appeal to causes at all. In fact, in many instances the various causal details need to be eliminated in

\footnotetext{
${ }^{1}$ This is the title of a paper by Alan Baker [1] who argues for an affirmative answer.

${ }^{2}$ In the context of the so-called indispensability debate, the acausal nature of mathematical entities is sometimes taken to be evidence against, either their existence, or our ability to know of their existence. See Colyvan [12, Chapter 3] for a discussion.
} 
order to gain genuine understanding of some phenomenon or other. ${ }^{3}$

Recent discussions of explanation in the philosophy of mathematics literature have focused on the indispensability argument. The idea is to try to show that mathematical entities are indispensable for physical theory in that they are indispensable for explanation. ${ }^{4}$ Many of the authors involved in this discussion readily admit that not all explanation is causal, and they debate the role of mathematical objects in noncausal explanation. [1, 12, 13] Some of these examples have been subject to criticism by Melia $[18,19]$. In response, Baker has attempted to provide a clear cut example where mathematical objects play an explanatory role.

Baker's example is interesting and persuasive. He argues that one can explain why the life cycle period of North American cicadas is a prime number of years (typically 13 and 17 years). Biologists have offered two evolutionary accounts (one involving the avoidance of predators and the other involving the avoidance of hybridization with similar subspecies). But both explanations appeal to facts about prime numbers and the number-theoretic notion of a lowest common multiple. [1, pp. 229-233]

Christopher Pincock, as well, provides a salient example of how a mathematical entity - a graph - with certain properties can play an explanatory role. His example concerns the inability to walk across all of the bridges in Königsberg returning to one's starting point and crossing each bridge only once. It is impossible to do so because the bridges and paths exhibit the structure of a non-Eulerian graph. [22, pp. 257-259] This is an example of a kind of abstract explanation - one that ignores (and requires that one ignores) various physical details about the system of interest and appeals to a particular abstract structure of the physical system. [22, p. 260] Many other examples, of abstracting explanations exist. I have argued [4] that what I call "asymptotic explanations" gain their explanatory power by the systematic throwing away of various causal and physical details. However, I disagree with Pincock's claim that asymptotic explanations are a subspecies of abstract explanations. [22, p. 260] At least I believe that, by and large, asymptotic explanations often do not proceed by focusing on an abstract structure realized by the physical system. This is important and I will discuss it in more detail below.

\footnotetext{
${ }^{3}$ See [4] for an extended discussion of how the elimination of (causal) detail can be explanatory.

${ }^{4}$ Baker [1] discusses various aspects of this argument.
} 
I have no criticisms to offer of either Baker's or Pincock's examples. Nor do I for most of the other examples of genuine mathematical explanation of physical phenomena one finds in the literature, e.g. [12]. However, I would like to point out one feature of both these examples and of most others in the literature: They are designed to show that some mathematical entity-a number or a graph (or some property of such an entity such as its primeness or the fact that it is or is not Eulerian) - plays an explanatory role. In other words, the aim of these examples is to show that mathematical entities, despite their abstract nature, can still be physically explanatory. I think that the reason for the focus on entities stems from the fact that these discussions all grow out of the debate about the indispensability of mathematics for natural science. The focus of that debate concerns reasons to believe in the existence of mathematical entities or objects. As a result, the authors have searched for examples demonstrating that mathematical entities are explanatorily indispensable. For instance Baker says that

[d]espite their opposing positions, Colyvan and Melia agree that establishing platonism stands or falls on whether specific examples can be found from actual scientific practice in which 'the postulation of mathematical objects results in an increase in the same kind of utility as that provided by the postulation of theoretical entities $^{5}$. [1, p. 224]

Here the "kind of utility" with which we are concerned is, of course, explanatory utility. ${ }^{6}$

On the other hand, there are, I believe, (many) examples of mathematical explanations of physical phenomenon that do not require that one associate a mathematical entity or its properties with some physical structure had by the system of interest. I present one of these in the next section. Following this, in section 4, I will discuss the philosophical conceptions of applied mathematics that have been proposed to account for the kinds of mathematical explanations we have been discussing.

\footnotetext{
${ }^{5}$ This latter phrase is from $[19, \mathrm{p} .75]$.

${ }^{6}$ Mary Leng argues that Colyvan's and Baker's examples can be explanatory even if the mathematical entities don't exist - that the explanatory utility of a mathematical model "does not depend upon the actual existence of the [mathematical] objects posited by the model." [16, p. 167] As will become obvious, my interests here lie elsewhere.
} 


\section{Mathematical Explanations II: Operations}

In contrast to explanations that appeal to mathematical entities (or properties of such entities) to explain physical phenomena, there are explanations which, while mathematical, do not make reference to such objects. Rather, these explanations appeal to (or better "involve") mathematical operations. I have discussed some examples in previous publications. [4, 5]

Consider the explanation offered by condensed matter physics for the so-called universality of critical phenomena. The explanandum is the remarkable similarity in behavior of "fluids" of different molecular constitution when at their respective critical points. ${ }^{7}$ Experimentally it is found that a certain dimensionless number - a critical exponent - characterizes the virtually identical behavior of diverse systems at their respective (and different) critical points. One would like to explain this remarkable universality of behavior or, in philosophical terms, how this multiply realized behavior is possible. ${ }^{8}$ Let me briefly introduce some of this terminology.

Consider the temperature-pressure diagram for a typical "fluid" in figure 1. The bold lines represents thermodynamic states in which two distinct phases of the fluid (pairs of solid, liquid, and vapor) can coexist. ${ }^{9}$ Thus, the lines represent states in which the system is undergoing a (first order) phase transition. For instance, along the line between points $A$ and $C$ one will find both liquid and vapor in the container - just what we see when the tea kettle boils on the stovetop. At point $C$, the critical point, something strange happens. Below the critical temperature $T_{c}$ and below the critical pressure $P_{c}$, the fluid exists in its vapor phase. Further, it is impossible, below $T_{c}$ and above $T_{a}$, to change the system from its vapor phase to the liquid phase by increasing pressure without crossing the line $A-C$. In other words, below $T_{c}$ the system cannot pass from the vapor to the liquid phase without going through a state in which both vapor and liquid are simultaneously present in the container. Above the critical temperature, $T>T_{c}$, it suddenly becomes possible to do this. Simply increase the temperature beyond $T_{c}$, increase the

\footnotetext{
${ }^{7}$ I put "fluids" in scare quotes because really there are many different kinds of systems that exhibit the same critical behavior including magnets and because, as we will see below, "fluids" can exhibit behavior that pre-theoretically we would not associate with fluids - i.e., liquids.

${ }^{8}$ See [3] for a discussion of the relationship between universality and multiple realizability.

${ }^{9}$ Point $A$ is actually a triple point - a state in which all three phases can coexist.
} 


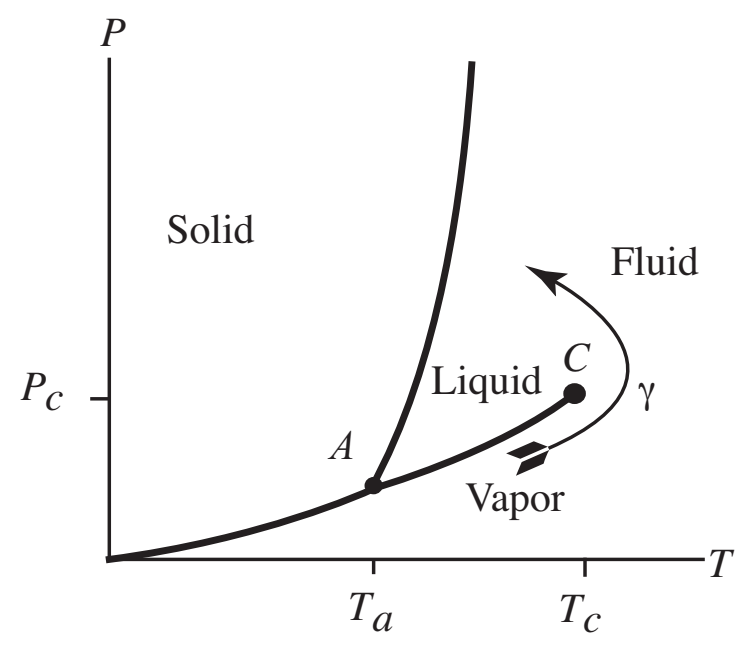

Figure 1: Temperature-Pressure Phase Diagram for a "Fluid"

pressure beyond $P_{c}$ and then decrease the temperature below $T_{c}$. This will be a path, $\gamma$, through states in which the "fluid" changes from vapor to liquid without ever being in a state where both phases coexist. The critical point indicates the existence of a qualitative change in the behavior of the system. Below $T_{c}$ the distinction between liquid and vapor makes sense; above $T_{c}$, it apparently does not. Thermodynamically, the qualitative distinction between different states of matter is represented by a singularity in a function (the free energy) characterizing the system's state.

Thus, mathematical singularities in the thermodynamic equations represent qualitative differences in the physical states of the fluid in the container. As mentioned above, at the critical point, systems with radically different microstructures exhibit behavior that mathematically is described by a particular number, the critical exponent $\beta$-the number is the same for the diverse systems. At the simplest level, the critical behavior can be characterized in terms of the behavior of a certain quantity, the so-called "order parameter," which for fluids is the difference in densities between the different coexisting phases. So along the line $A-C$ in figure 1 the order parameter, $\Psi$, is the difference between the liquid and vapor densities:

$$
\Psi=\left|\rho_{\text {liq }}-\rho_{\text {vap }}\right| .
$$

Below the critical temperature, $T_{c}, \Psi$ is nonzero indicating the simultaneous 


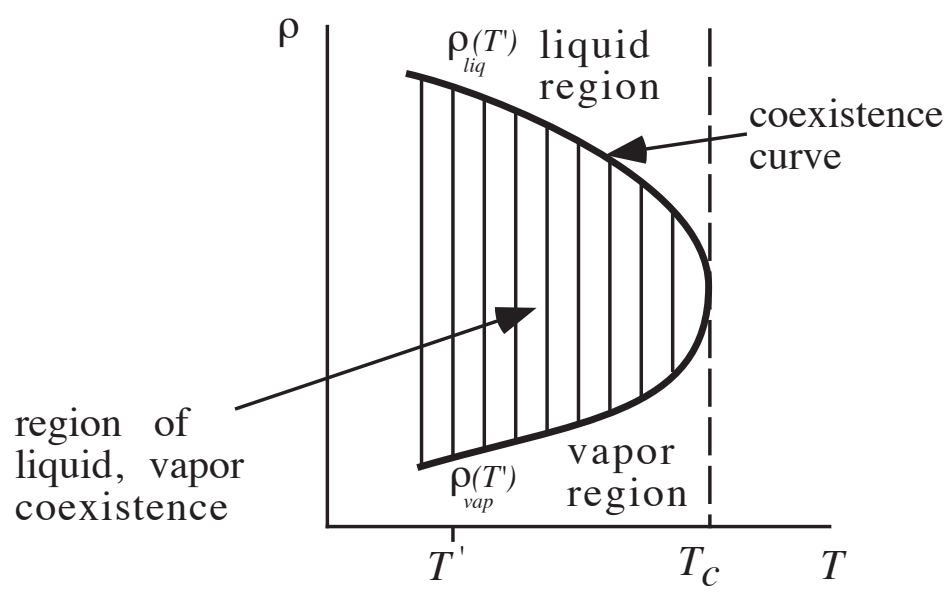

Figure 2: Coexistence Curve: Density vs. Temperature for a "Fluid"

existence of both liquid and vapor in the container. Above $T_{c}$, the order parameter takes the value zero. Figure 2 exhibits the vanishing of the order parameter $\Psi$ at $T_{c}$. The heavy line is the coexistence curve with the vertical "tie lines" indicating that at some specific temperature $T^{\prime}$, the liquid density is $\rho_{\text {liq }}\left(T^{\prime}\right)$ and the vapor density is $\rho_{\text {vap }}\left(T^{\prime}\right)$. Note that at $T_{c}$ the difference between these two values vanishes.

One universal feature-a feature displayed by all of the distinct fluids at their respective critical temperatures - is the fact that the coexistence curves near each of these $T_{c} \mathrm{~s}$ have the same shape. We can introduce a "distance" measure called the "reduced temperature", $t$, that allows us to describe how far any system is from criticality. This allows us to compare the critical behavior of different systems with different $T_{c} \mathrm{~s}$ - different critical temperatures. The reduced temperature is

$$
t=\left|\frac{T-T_{c}}{T_{c}}\right|,
$$

and the universality claim amounts to the fact that the order parameter, $\Psi$, for every fluid vanishes as some power $\beta$ of $t$ :

$$
\Psi=\left|\rho_{\text {liq }}-\rho_{\text {vap }}\right| \propto t^{\beta} .
$$

The number $\beta$ characterizes the shape of the coexistence curve in the neighborhood of $T_{c}$. For instance, if the curve is a parabola, then $\beta$ would be 
$1 / 2$ - a result erroneously predicted by mean field theory. Experimentally, it has been found that the $\beta$ is not $1 / 2$ but rather some number close to 0.33 .

The explanatory question then is why the order parameters for various physically distinct fluids (and even magnets where the order parameter is the net magnetization) scale as a specific power law $t^{\beta}$. Condensed matter theorists have provided an explanation for this fact that involves the so-called renormalization group. ${ }^{10}$ Without going into detail here, one essential feature of the explanation provided is the invocation of the so-called thermodynamic limit. This is the limit in which (roughly speaking) the number of particles of the system, e.g., the number of $\mathrm{H}_{2} \mathrm{O}$ molecules in the tea kettle, approaches infinity. And, of course, this is an idealization: water in real tea kettles consists of a finite number of molecules. This limiting idealization is essential for the explanation because for a finite number of particles the statistical mechanical analogs of the thermodynamic functions cannot exhibit the nonanalytic behavior necessary to represent the qualitatively distinct behaviors we observe. ${ }^{11}$ Kadanoff puts it as follows:

The existence of a phase transition requires an infinite system. No phase transitions occur in systems with a finite number of degrees of freedom. [15, p. 238]

The claim that the explanation of the existence of phase transitions and of the universality of critical phenomena requires the thermodynamic limit (that the limiting idealization is explanatorily essential) is not uncontroversial. Some, like Craig Callender [11], argue that to accept this is to "take thermodynamics too seriously." I've argued in [5] that, to the contrary, on this point we should take thermodynamics very seriously indeed. ${ }^{12}$ But the main point here is that if I am right, and taking the thermodynamic limit is an explanatory essential mathematical operation, then this is a case in which, while we have a genuine mathematical explanation of physical phenomenon, there is no appeal to the existence of mathematical entities or their properties. Instead, the appeal is to a mathematical idealization resulting from a limit operation that relates one model (the finite statistical mechanical model) to another (the continuum thermodynamic model).

\footnotetext{
${ }^{10}$ See [15] for an account by one of the founders of the technique and [4, chapter 4$]$ for more a more philosophical discussion.

${ }^{11}$ For an extended discussion of essential or "ineliminable" idealizations in physics see [14]. See also [5] for related work.

${ }^{12}$ See also, [2].
} 
There are other examples of this type of mathematical explanation. In fact, I believe that most idealizations in applied mathematics can and should be understood as the result of taking mathematical limits. In [4], for example, I wrote extensively about how one can explain the fact that the spacings and intensities of the bows in rainbows are the same, despite the fact that the (causal) details of how each rainbow gets formed will be completely distinctfor instance, the sizes and shapes of the raindrops will vary from rain shower to rain shower. The explanation in this case also involves the taking of a limit: In order to explain the universal pattern we witness in rainbows, we need to examine the wave theory of light in the limit as the wavelength goes to zero. When the wavelength equals zero, we are in the domain of ray theory or geometrical optics and it turns out that stability properties of ray-theoretic structures explain the universal rainbow pattern that we witness. [4, chapter 6] (See also $[9,6]$.) The important thing, again, is that the limiting operation between models leading to the idealized description in terms of rays plays an ineliminable role in the explanation of the physical pattern that we witness.

I will argue below that the "standard philosophical accounts of applied mathematics" are unable to account for the role of limiting idealizations in mathematical explanation. As a result, a completely new approach to the applicability of mathematics is required.

\section{Mapping Accounts: Strengths}

What, then, are the "standard philosophical accounts of applied mathematics"? Given the relatively recent interest in the topic of the applicability of mathematics, it isn't clear that this is entirely a well-formed question. On the other hand, recent investigators do seem to have an approach to applicability on which, in broad outline, they agree. Bueno and Colyvan [10], following Pincock [22], call such accounts "mapping accounts." ${ }^{13}$ In a nutshell mapping accounts seek to explain the utility of mathematics in some applied situation by demonstrating the existence of the right kind of map from a mathematical structure to some appropriate physical structure.

Pincock [22] address the issue of how to interpret statements that arise in applied mathematics. He calls these "mixed statements," and holds that

\footnotetext{
${ }^{13}$ Bueno and Colyvan are at pains to argue that Pincock's mapping account cannot be the full story about the applicability of mathematics, and while I agree, I don't believe Pincock ever made such a strong claim.
} 
what all mixed statements have in common is "the occurrence of what seems to be mathematical terms along with nonmathematical terms." [22, p. 137] An example of such a mixed statement is "the mass of the satellite is 100 kg." Pincock's proposal is that one can provide truth conditions for mixed statements by providing an appropriate mapping from the mathematics to physical situation. A very simple example is how to understand the mixed claim: "There are 4 apples on the table." [22, p. 145] On the mapping (or "structuralist") account favored by Pincock, this sentence comes out true just in case "there is an isomorphism from [apples] to an initial segment of the natural numbers ending with [4]." [22, pp. 145-146]

Pincock's appeal to the non-Eulerian nature of certain graphs to account for path restrictions on walking the bridges of Königsberg is another example that fits with the mapping account. Here there is a mapping from the graph structures in the mathematical domain to the structural relations between the actual bridges in Königsberg. Certain relations between parts of the bridge system allow for a mapping between an appropriate graph and those parts. In this case, as I've noted above, it seems that the existence of this mapping enables us to provide a mathematical explanation of the physical path restrictions; though, as Pincock rightly emphasizes, the explanatory feature results from the abstraction involved in highlighting the formal relational structures of the physical (bridge) system. [24, p. 257-259]

The point I wish to emphasize here is that underlying both the purely representative aspects of (the mixed statements of) applied mathematics, and the explanatory aspects, is the idea that the proper understanding of applied mathematics involves some sort of mapping between mathematical structures and the physical situation under investigation. While they criticize Pincock's position in various ways, Bueno and Colyvan [10], as well, endorse the position that a proper account of the applicability of mathematics is to be grounded in some kind of mapping between the mathematical domain and the physical.

For instance, Bueno and Colyvan, in presenting what they call an "inferential conception of the application of mathematics," see themselves as extending or building on the "pure" mapping account they attribute to Pincock. $^{14}$ They say

\footnotetext{
${ }^{14}$ I think Bueno and Colyvan are a bit uncharitable in their interpretation of Pincock. They claim that according to Pincock "the existence of an appropriate mapping from a mathematical structure to a physical structure is sufficient to fully explain the particular application of the mathematical structure in question." [10, p. 1] I don't see Pincock as
} 
$[w]$ hy is it that mathematics is so useful in empirical science? One answer is simply that mathematics is a rich source of structures and therein lays its utility. Some mathematical structure is either designed to, or otherwise found to, accurately capture the important structural relations of an empirical set up, and we can thus read off important facts about the empirical set up from the mathematics. [10, pp. 1-2]

Further, they argue that

[t]here is clearly something right about the mapping account. Mathematics is a rich source of structures and when some mathematical theory finds applications in empirical science, it is clear that the mathematics captures certain important structural relations in the system in question. [10, p. 3]

I believe that all of these claims are correct. Surely something is right about the mapping account. In particular, when it comes to representing physical structures, mathematical structures often provide useful models that abstract (as Pincock stresses) from various explanatorily irrelevant physical details. My disagreement, as will become evident, concerns the necessity of representation for explanation. Instead, what is often explanatorily essential is the mediating limiting relationship between the representative models. To put this slightly differently, mapping accounts focus on "static" relationships between mathematical models and the world. My view is that this misses, in many cases, what is explanatorily relevant about idealizations; namely, that they often involve processes or limiting operations.

\section{Mapping Accounts: Idealizations}

Many explanatory models appear to involve idealizations. We speak of frictionless planes when there are no such things, and we idealize fluids to be continua when, in fact, they are composed of discrete finite collections of molecules. If we accept that idealizations can and do play important roles (perhaps even explanatory roles), then that raises a deep problem for mapping accounts of the applicability of mathematics. The problem is simple:

saying anything a strong as this sufficiency claim. On the other hand, I believe all of these investigators endorse the idea that the right kind of mapping is necessary for explaining any particular application of mathematics to the physical world. 
Nothing in the physical world actually corresponds to the idealization. So in what sense can we have a mapping from a mathematical structure to an existing physical structure? Mapping accounts are representative and good representations reflect the truth about the world. Idealizations, however, are false.

Both Pincock, and Bueno and Colyvan, are well-aware of the difficulties mapping accounts will have with idealizations: Since idealizations are necessarily false of the physical world, there can be no physical structure to be mapped onto an appropriate mathematical structure. Despite their differences, both, it seems to me, try to deal with this problem in a similar way. In the next two subsections I will discuss their proposals. Following that in section 6 I will argue that neither attempt to allow for certain types of idealizations succeeds.

\subsection{Pincock and Matching Models}

A natural hallmark of mapping accounts of the applicability of mathematics is that such maps provide representations of physical structures using mathematical machinery. Pincock is explicit in holding that "[m]athematical idealizations are scientific representations that result from assumptions that are believed to be false, and where mathematics plays a crucial role." [23, Abstract] Pincock recognizes the immediate problem with this view of mathematical idealization. He asks:

What guarantee is there that the results of employing these false assumptions will be representations? Or, more precisely, as representations may be ranked in terms of their accuracy and adequacy, why should making false assumptions contribute to the production of good representations? [23, pp. 958-959]

Pincock proposes to respond to these questions by providing an account of how one can in fact rank idealizations (with their false assumptions) as better or worse for representing the world.

Pincock's example is the idealization in which a discrete equation characterizing Newton's law of cooling - an equation that describes the amount of heat per unit time passing from a warmer plate 2 to a cooler plate 1 - is replaced by the more idealized one dimensional heat equation, a partial differential equation that treats the discrete quantities as continua. The discrete 
equation is:

$$
\frac{\Delta Q}{\Delta t}=\frac{\kappa A}{d}\left|T_{2}-T_{1}\right|
$$

Here $\Delta Q$ is a discrete quantity of heat, $\kappa$ is the thermal conductivity of the material, $A$ represents the area of the plates, and $d$ is their distance from one another.

The continuum heat equation for $u(x, t)$, the temperature at a point $x$ at time $t$, is:

$$
\frac{\partial}{\partial t} u(x, t)=\frac{\kappa}{\rho s} \frac{\partial^{2}}{\partial x^{2}} u(x, t) .
$$

Here, $\kappa$ is as above, $\rho$ is the material density and $s$ is its specific heat.

Consider the heat equation (2) as our idealized "equation model" (to use Pincock's terminology). The question is how this obviously false idealized equation can be used to represent heat transfer? Pincock holds that an equation model such as (2)

represents a physical situation when the scientists believe both that (i) there is an isomorphism between the physical situation and a matching model and (ii) there is an acceptable mathematical transformation between the equation model and the matching model. The matching model contains wholly mathematical analogues of all the physical magnitudes in the physical situation. A mathematical transformation will be acceptable when it is consistent with the goals of the scientists in terms of scale and accuracy. [23, pp. 962-963]

The matching model is a model that is supposed to perfectly reflect the physical situation of interest. Thus, every physical parameter (relevant or otherwise) of a given system will find a counterpart in the matching mathematical model. A natural reading of this would be that a matching model provides an absolute fixed point (like $0^{\circ}$ Kelvin for the temperature scale) with respect to which we can judge the representativeness of one model to another - the closer it is to the matching model the more representative and, hence, better it is.

I think that when it comes to assessing the overall "representativeness" of a model, Pincock must be committed to something like this reading - the closer the equation model is to the matching model, the more representative it will be. However, he explicitly denies that for an idealization to be good one requires a global metric or distance measure between the equation 
model and the matching model. On his view, one can judge locally an idealization/representation to be good to the extent that it meets the "scale and accuracy" (the experimental error) acceptable to scientists. This will, apparently allow for a nonabsolute measure of the goodness of idealizations. Thus, this view is compatible with having conflicting (possibly equally good) idealizations and, therefore, fits with Morrison's discussion of the role of idealization in physical theories. [20]

There is a tension for Pincock in these assertions. On the one hand, he wants to present an account of idealizations that allows one to rank idealizations, yet at the same time he denies a global measure of distance between the full representation (the matching model) and one's idealized equations model. Local measures of goodness, do not, as far as I can tell, allow for comparative rankings of different idealized models. I believe this tension becomes acute in the context of trying to develop an account of how idealizations can be explanatory and I will return to this below. Prior to this, let me discuss Bueno's and Colyvan's proposal.

\subsection{Bueno, Colyvan and the Inferential Conception}

In [10] Bueno and Colyvan propose an inferential conception of the application of mathematics. They explicitly note that it is an extension of the mapping account proposed by Pincock in that it includes features that are not purely structural. "Unlike the mapping account ... the proposal advanced here is not purely structural, since it makes room for additional pragmatic and context-dependent features in the process of applying mathematics." 15 $[10$, p.7]

The crucial feature of the proposal is that the fundamental role of applied mathematics is inferential: by embedding certain features of the empirical world into a mathematical structure, it is possible to obtain inferences that would otherwise be extraordinarily hard (if not impossible) to obtain. ... [Mathematics has other roles, from] unifying disparate scientific theories through helping

\footnotetext{
${ }^{15}$ As we have just seen in the last section, however, Pincock's proposal (at least when it comes to dealing with idealizations) is not purely structural either. He appeals to pragmatic features and context when claiming that the equation models is to be judged relative to the matching model in terms of a measure of scale and accuracy that is acceptable to scientists. [23, p. 963]
} 


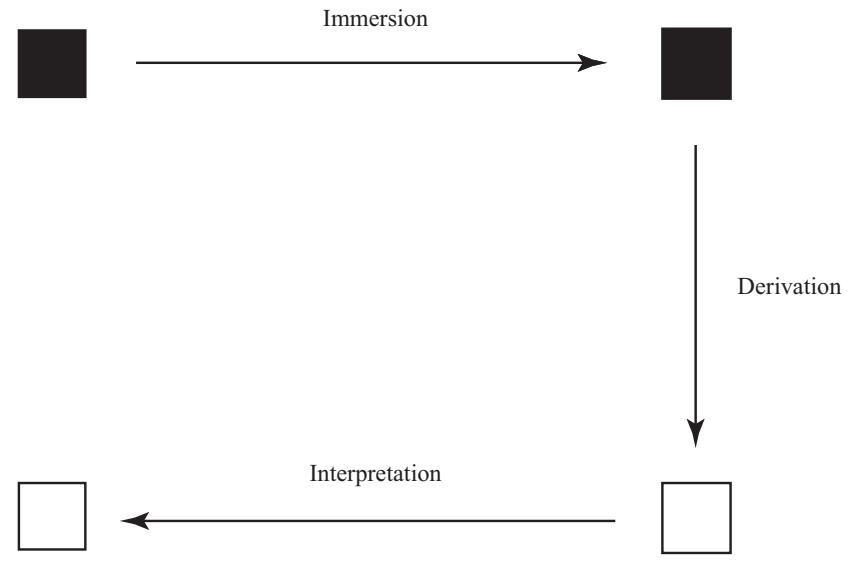

Figure 3: Inferential Conception of Applied Mathematics [10, p. 9]

to make novel predictions (from suitably interpreted mathematical structures) to providing explanations of empirical phenomena (again from certain interpretations of the mathematical formalism).

All of these roles, however, are ultimately tied to the ability to establish inferential relations between empirical phenomena and mathematical structures, or among mathematical structures themselves. [10, p. 7]

Their inferential conception involves two mappings:

- An "immersion" map from the physical system or "empirical set-up" to an appropriate mathematical structure.

- An "interpretation" map back from some (possibly new) mathematical structure to the physical system or empirical set-up of interest.

In between these two mapping relations there is what they call a "derivation step" in which inferences are drawn about the mathematical structures purely from within mathematics. See figure 3.

How do Bueno and Colyvan attempt to accommodate idealizations in this framework? To do this they appeal to an apparatus of partial mappings. I do not believe that I need to go into detail about how this apparatus is supposed 
to function. [10, p. 13-14] One can get a feel for what partial mappings are supposed to do by briefly examining the examples they cite.

They consider the case of neoclassical economics where agents are modelled as being perfectly rational in that they maximize their expected utility functions. Since real world agents are not perfectly rational in this sense, such a representation is an idealization. As they say,

$[T]$ here's no full mapping between the behavior of agents in the actual world and the ... mathematical structures of [analysis]. After all, actual economic agents do not necessarily maximize their utility functions .... In this case, we have at best partial mappings between the behavior of actual economic agents (corresponding to features that actual agents try to preserve in practice) and the relevant mathematical structures. [10, p. 14]

Despite this Bueno and Colyvan point out that

[t]his doesn't mean that neo-classical economics has nothing to offer. As even critics of neo-classical economics should be able to recognize, there are partial mappings between certain aspects of the actual economic scenario .... and the mathematical model .... This indicates in which respect neo-classical economics, although idealized, can still say something about the world, albeit indirectly. There are aspects of the actual world - although certainly not every aspect - that are successfully captured by the relevant models. Even in idealized contexts there are partial mappings between empirical and mathematical structures. [10, pp. 15-16]

At this point, one might ask why simply having a partial mapping between some aspects of the physical situation and an appropriate mathematical structure accounts for the explanatory role that idealizations can play in applied contexts. So far what we have is a framework in which we can get some kind of partial representation of the full actual structure. (This partial representation is, I believe, to some extent analogous to Pincock's equation model discussed in section 5.1.) Prima facie, it seems we have no reason to believe that simply having an appropriate (partial) mapping is explanatory. Indeed, what is the argument that such a partial representation itself plays an explanatory role?

While not explicit in their paper I think we can get some idea of the kind of answer Bueno and Colyvan might want to give to this question. (It is similar 
to what I take to be Pincock's response to the same problem.) They discuss another example from economic theory - Herbert Simon's theory of bounded rationality. On this account, rational agents do not strive for anything as strong as the maximization of some utility function; rather they, search for a "satisficing" alternative. This view explicitly

emphasizes two basic sort of limitations involved in actual decision making in economics: (i) limitations on the economic agent: he or she has limited computational and cognitive power; and (ii) limitations on the nature of information about the environment: often, the agent has at best incomplete information about alternatives. $[10$, p. 16$]$

Bueno and Colyvan note that this theory is a significant improvement over the neoclassical theory just discussed. But they point out that despite its less idealized status, "... there will be at best partial mappings between the actual economic situation and the new mathematical models, given that the economic situation is now thought of as fundamentally incomplete." $[10, \mathrm{p}$. 17]

They summarize the discussion as follows:

... it now becomes clear that, also in Simon's case, partial isomorphisms or partial homomorphisms are central in the immersion step. These mappings allow us to move from the limitations of the empirical set up (the partiality of information that agents have and their limited cognitive capacities) to the appropriate mathematical models. Similarly, after derivations are obtained, the interpretation step can also be implemented with partial isomorphisms or partial homomorphisms. After all, ... the empirical set up is now characterized by partial information, and so only partial mappings will hold from the mathematical model back to the empirical set up. In the end, partial mappings are crucial even when we deal with less idealized models, such as developed by Simon. [10, p.18.]

It seems reasonable to assume from this discussion that Bueno and Colyvan consider Simon's less idealized model in an effort to demonstrate the possibility of finding more realistic models that improve upon those that are more idealized. I think this fits with Pincock's desire to rank idealizations as better (more representative) than others. Thus, (and this is not explicit in Bueno and Colyvan) perhaps part of the answer to why some idealizations 
can play explanatory roles, despite being false, is because we can tell a story about how they ultimately can be removed by paying more attention to details that are ignored or overlooked by more idealized models: If we only had a more complete characterization of the actual economic situation, we would be able to approach (in some measure) a full isomorphism between the world and the mathematical model. So, in the end, the partial mappings, that are required because of idealizations, can play explanatory roles because one expects that their partialness can be (in principle) eliminated yielding a full isomorphism - a complete representational mapping.

However, this suggestion for what might make an idealization provided by a partial mapping explanatory is not endorsed by Bueno and Colyvan. Neither, as we've seen, is it endorsed by Pincock. Pincock denies the existence of such a global measure. Colyvan, as well, in a personal communication, says that he does not intend the discussion to suggest that the less idealized model (Simon's satisficing account) will necessarily be more explanatory than the more idealized model. So the tension present in Pincock's proposal exists for Bueno's and Colyvan's account as well: Partial representation enables a ranking of idealizations in terms of representativeness but that ranking does not correlate with a given idealization being more explanatory than another.

There are two desiderata at play. First, one would like mapping accounts to allow idealizations to be explanatory. Second, one would like (explicitly for Pincock) to rank idealizations in terms of their representational goodness. A natural way to satisfy both of these desiderata would be to adopt a Galilean understanding of idealizations of the sort advocated by McMullin [17] and others. This is a view in which idealizations are perfectly respectable in science, provided that one can (in principle) tell a story about how they might be removed through further work. However, neither Pincock, nor Bueno and Colyvan want to go down that road. I think this puts them in a difficult situation as mapping accounts that meet the two desiderata seem to require such a view.

\section{Mapping Accounts: Limitations}

We have seen that Pincock, as well as Bueno and Colyvan, endorse some form of mapping account of the applicability of mathematics. As a result both are forced to consider some relationship between mappings that represent more and less idealized situations. But let me ask again the question I 
raised earlier: How does having a representation or a partial representation of a physical situation in mathematical terms provide an explanation of that physical situation?

In some contexts, the answer appears to be the promise of being able to tell an additional story about how the representation can be improved-how the idealizations can be de-idealized. Such a story fits well with what I have elsewhere called the "traditional view" of idealization. [8] A better name, following McMullin's seminal contribution would be "Galilean" idealization. [17] The guiding idea is somewhat paradoxical: Idealizations are compatible with good science (both descriptively and explanatorily) to the extent that they can be eliminated through further work that fills in the details ignored or distorted in the idealized model.

There are many situations in which this Galilean conception of idealization is appropriate. And, to the extent that it is, I believe accounts of the applicability of mathematical idealizations in natural science can be understood in terms of mapping accounts. A very helpful, and often quite accurate way of understanding this mathematically is in terms of regular (analytic/nonsingular) perturbation series: The idealization is the first order term in the series and we improve upon that by adding correction terms in powers of the relevant parameter. (This is the natural way of making the concept of a de-idealizing story mathematically precise.)

However, it is worth noting again, that neither Pincock, nor Bueno and Colyvan want to adopt this Galilean conception. They both want to allow for "good" (and explanatory?) idealizations without having to commit themselves to the kind of global ranking that would enable them to tell the required de-idealizing story. And, once again, given that mapping accounts seem almost to require a representational ranking, this rejection of the Galilean picture does not fit well.

Despite this, however, I think that far more often interesting idealizations are not de-idealizable. I think that there are many cases of "nontraditional" idealization in which idealizations actually play an essential, ineliminable role in explanatory contexts. ${ }^{16}$ (This may be the reason Pincock, and Bueno and Colyvan, want to resist the traditional or Galilean picture.) Of course, we have already seen one such example in section 3. There we saw that the thermodynamic limit plays a crucial and ineliminable role in understanding the universality of critical phenomena. Other examples, are discussed in [8],

\footnotetext{
${ }^{16} \mathrm{By}$ "ineliminable" here I mean that no de-idealizing story is possible even in principle.
} 
[5], and [4].

Nontraditional idealizations do not (and cannot) have any promissory background story about how to fill in the details. In fact, such idealizations trade on the fact that in many instances "overly simple" model equations can better explain the most salient features of a phenomenon than can a more detailed less idealized model. (Again, this comports well with Pincock's and Bueno's and Colyvan's intuitions about idealizations, but is ultimately, I believe, at odds with structuralist/mapping accounts of applicability.) ${ }^{17}$ On the nontraditional conception, adding more details counts as explanatory noise - noise that often obscures or completely hides the features of interest.

Nontraditional idealizations cannot provide such a promissory background story because the limits involved are singular. This is, in fact, the case in the renormalization group explanation of the universality of critical phenomena. (Here, the perturbation problem is singular and there is no convergent series expansion such as one finds in regular/analytic perturbation problems.) A singular limit is one in which the behavior as one approaches the limit is qualitatively different from the behavior one would have at the limit. A simple example (overly simple in some ways) is the following. Suppose we have a quadratic equation

$$
x^{2}-\epsilon 2 x-2=0
$$

where $\epsilon$ is a parameter. Consider the limit $\epsilon \rightarrow 0$. For any nonzero value of $\epsilon$ the quadratic equation has two roots. And, in fact, at $\epsilon=0$, the solution set also has two roots; namely, $+\sqrt{2}$ and $-\sqrt{2}$. So there is a sense in which the qualitative behavior of the solution set doesn't change when one reaches the limit of zero: There remain two roots throughout the limiting operation.

Had the parameter multiplied the quadratic term, the situation would be radically different. In this situation the equation is

$$
\epsilon x^{2}-2 x-2=0
$$

and as $\epsilon \rightarrow 0$ there are will be two roots for any nonzero value of $\epsilon$. However, at $\epsilon=0$ there is only one solution: $x=-1$. A qualitative change in the structure of the solution set has occurred. In this case, (speaking somewhat but not completely metaphorically) it isn't possible to have a "measure" of how close the $\epsilon=0$ solution is to any $\epsilon$ is-very-very-small solution.

\footnotetext{
${ }^{17}$ At the very least, proponents of mapping accounts that reject the Galilean story owe us an account of how a mere partial representation can be explanatory.
} 
In the thermodynamic limit, as it is employed in the explanation of the universal scaling of the order parameter for critical systems, there emerges a host of divergences and singularities. Crucial among these is the divergence of the so-called correlation length. This implies a loss of any sort of characteristic length scale and allows the various systems to be compared with one another asymptotically. Such a loss of scale is required to demonstrate the genuine qualitative change in the states of matter that occur at criticality. Finite systems with more and more particles may in some sense ${ }^{18}$ get "close to" the nonanalytic behavior in the thermodynamic functions, but for finite $N$, the curves are always smooth. There is no distance measure or metric saying how close an actually smooth curve is to a nonsmooth/nonanalytic one. (Again, the related perturbation problem is singular.)

There are two important and related features of nontraditional idealizing explanations evident in this example. First, the explanation for the universality of critical phenomena requires singularities; in particular, the divergence of the correlation length. Without this, we have no understanding of how physically diverse systems can realize the same behavior at their respective critical points. Second, singularities make it impossible to tell any kind of de-idealizing story that would enable one to rank idealizations in terms of their distance from a matching model (Pincock) or from full isomorphism (Bueno and Colyvan).

One consequence of this is that the kind of explanations provided by the renormalization group (and numerous other examples of the use of limiting asymptotic idealizations for explanatory purposes) are not representative. Some mathematical explanations of physical phenomena appeal to limiting operations and these are simply not the sorts of gizmos which figure in a (partial) representation, the explication of which is the aim of the various mapping accounts.

Another slightly different way to see this is by noting that there are no structures (properties of entities) that are involved in the limiting mathematical operations. That is, limiting mathematical operations typically do not yield anything like the abstract non-Eulerian structure of the bridge system in Pincock's example. If the limits are not regular, then they yield various types of divergences and singularities for which there are no physical

\footnotetext{
${ }^{18}$ For instance, the relevant curves "look" sharper and sharper as the number of particles increases. But "looking sharp" is not a relevant measure: For any finite N, no matter how large the curves are smooth and analytic, no matter how sharp they appear.
} 
analogs. Nevertheless, as we saw in section 3 these singularities are essential for genuine explanation. One might, I suppose, stretch terminology a bit and call the various divergences "structures", but this won't help the mapping theorists as there are no possible physical structures analogous to such mathematical "structures". 19

In sum, if nontraditional idealizations do play explanatory roles in physical theory, then mapping accounts simply cannot be the whole story about the explanatory applicability of mathematics in natural science. Some new story needs to be told in which limit operations (rather than mathematical entities and their properties) are important supporting characters and where the mathematical singularities that emerge in those limits play starring roles. In the next section I begin to outline what such a tale should look like.

\section{Suggestions for a New Approach}

The mapping approaches to the applicability of mathematics emerged out of a concern with the indispensability problem. ${ }^{20}$ This motivation, as I noted in section 2, was to find a role in physical theory for entities that are abstract and acausal. One could say, fairly I think, that these approaches originate from a concern with the nature of mathematical entities (and our ontological commitments to them). They are not driven by considerations of physical application in any direct way.

I have been arguing that there is another aspect to the applicability problem; namely, to account for the role played by limiting idealizations in applied mathematics. I think the proper approach to this should come from the side of physics (and not from philosophical concerns about the nature of abstract entities). The guiding question becomes: Does the physical world dictate in any way the kind of mathematics that must be used to explain and understand physical phenomena? I believe the answer to this question is "yes" and that as a result we can formulate a new account of the role of mathematics in physical theorizing. The rest of this section provides a brief discussion of

\footnotetext{
${ }^{19}$ This is my response to Pincock's claim that asymptotic explanations are a subspecies of what he calls abstract explanations. See [22, p. 18] and the brief discussion above in section 2 .

${ }^{20}$ I should say "contemporary mapping approaches." There is indeed a long history of trying to understand the relationship between the axioms of geometry, theories of space, and formal logic. See, for instance, [21].
} 
how the answer to this question can be in the affirmative. ${ }^{21}$

Begin by noting that most (though not all) explanations in physics and applied mathematics are explanations of patterns or regularities. ${ }^{22}$ The universality of critical phenomena is an example of a surprising patterndifferent fluids all display identical behavior at criticality. Another example, mentioned at the end of section 3 concerns why rainbows always appear with the same bow structure - the same pattern of intensity and spacings of the colored and dark bands. Each rainbow is the result of a unique set of circumstances. The wind will be different, the raindrop shapes and sizes can vary considerably, etc. Nevertheless, despite the fact that various details are completely distinct, we witness the same pattern. We would like an explanation of why. (See [4, Chapter 6].) In fact, any phenomenon that we see repeat itself at various times and locations - any repeatable or reproducible experiment - will always occur in situations with different details. The world is a complicated place and is continually changing in many ways.

In order to explain the robustness (the repeatability) of the patterns we see, our mathematical representations have to be stable under changes of all these different details. One important way mathematics allows us to do this is through the taking of limits. Limits are a means by which various details can be thrown away. (For instance, in taking the thermodynamic limit in the context of explaining fluid behavior, we eliminate the need to keep track of individual molecules and we remove details about the boundaries of the container in which the fluid finds itself, etc.) In applied mathematics this taking of limits is sometimes called "variable reduction" and it often has interesting consequences. For instance, in taking such limits we are often led to focus on mathematical singularities that can emerge in those limits. The divergence of the correlation length in the renormalization group explanation of the universality of critical phenomena is one such emergent singularity.

In the context of the rainbow patterns, we are in the realm of the wave theory or wave optics where lightwaves are governed by a wave equation. When we investigate the nature of this equation in the limit in which the wavelength of light approaches zero, we might expect to smoothly obtain ray or geometrical optics - the theory in which light is composed of rays rather

\footnotetext{
${ }^{21}$ This is only a sketch of a full answer. Further elaboration is obviously needed and is the topic of current investigation.

${ }^{22}$ We do at times care about understanding sui generis behavior like transients in a circuit before it settles down to a steady state. But mostly, we are interested in understanding patterns that present themselves to us at different times and different places.
} 
than waves. However, such a smooth limit does not exist. ${ }^{23}$ There are various mathematical blow-ups and singularities in the zero wavelength limit. (One should, for example, expect light of infinite intensity on the emergent focal surfaces or caustics.) Nevertheless, the asymptotic investigation of this equation is essential for an understanding of why rainbows always appear with the same patterns of intensities and spacings of their bows. [4, Chapter 6] The asymptotic investigation of the wave equation leads to an understanding of the stability of those phenomena under perturbation of the shape of raindrops and other features. Stability under perturbation of details is exactly what is required for a phenomenon to be repeatable or reproducible.

It is fair to say, however, that most philosophical discussions of explanation in the natural sciences eschew places where the mathematics (via the development of singularities) would say that the world isn't law-governed. That is, explanations most always involve some kind of subsumption of the explanandum phenomenon under some kind of regularity . (Even sophisticated non-covering law accounts, such as Woodward's [28] recent causal account, look to invariances - kinds of regularities - of some kind or another.) But if ones interest is in understanding the robustness of the patterns of behavior that we see, a focus on regularities and lawlike equations very often turns out to be the wrong place to look! We need to understand why we have these regularities and invariances. We need, that is, to ask for an explanation of those very regularities and invariances. This is the fundamental explanatory question. The other accounts don't ask that question, in that they typically treat those regularities and invariances as given. The answer to this fundamental question necessarily will involve a demonstration of the stability of the phenomenon or pattern under changes in various details.

Remarkably and paradoxically, many applied mathematical accounts of the robustness of patterns - of the repeatability of phenomena despite wide variations in various details - involve the investigation of singularities. To explain and understand the robustness of patterns and regularities one sometimes needs to focus on places where those very regularities breakdown. Thus, the existence of patterns or regularities in the world and our desire to understand and explain them, has led us rather straightforwardly to investigate singularities in mathematical limiting operations. I suggest that this is one

\footnotetext{
${ }^{23}$ Why would we be interested in this limit? Because, the wavelength of light is much smaller than any other distance parameter in the problem such as the diameter of a raindrop. This directs us to the asymptotic shortwave limit.
} 
way to begin to understand the effectiveness of mathematics in applied situations. Furthermore, it is an approach that is completely orthogonal to structuralist/mapping accounts that take explanations necessarily to involve static representational maps.

In recent work, I have focused on some examples of this odd but ubiquitous situation [5, 7]. Mark Wilson, in a slightly different context, argues for a similar point of view about the importance of singularities. His discussion [26, p. 189-190] of shock waves in a gas is representative: Imagine we have a tube full of a gas and we blow in one end. Considered as a collection of molecules, if the initial blowing is hard enough, then fast molecules will eventually pass slower ones further down the tube-just like a traffic jam on a highway. If, however, we model the gas in the continuum limit as a (continuous) fluid, the mathematics will develop a singularity - the model will say, for example, that the gas should have two different velocities at the same place and time. Wilson notes "[p]rima facie, one would expect that this apparent contradiction in the mathematics will force us to abandon our smoothed out fluid description ...." [26, p. 189] But rather than move back to a description of the gas as a finite collection of molecules we can sweep all of those details under the rug and consider the mathematical shockthe singularity - as a boundary that dominates the physics of the situation. That singular surface constrains and largely determines the behavior in the law governed regions on either side of the shock. ${ }^{24}$

Wilson says that

... from a modeling point of view, we should object to the appearance of singularities where some density or velocity blows up to infinity; from a mathematical point of view, we often greatly value these very same breakdowns, for ...the singularities of a problem commonly represent the precise features of the mathematical landscape we should seek in our efforts to understand how the qualitative mathematics of a set of equations behaves. Insofar as the project of achieving mathematical understanding goes, singularities prove our best friends, not our enemies. ([27, p10]

I understand his conception of a "modeling point of view" to be a "representative point of view." Indeed such mathematical blow-ups are not always

\footnotetext{
${ }^{24}$ Wilson [26, p. 362] calls this "physics avoidance": "the policy for reducing descriptive variables through compressing behavioral complexities into boundaries and singularities ..."
} 
representative of physical systems.

Consider another example. When water drips from a faucet, it appears to undergo a genuine physical discontinuity - one topological blob breaks into two. And if we describe the drop using continuum hydrodynamics, there are mathematical singularities that develop in finite time, corresponding to the breaking of the drop. ${ }^{25}$ However, at the molecular level, no such singularity exists. We can follow the individual molecular dynamics and will never be able to identify the place and the time where the drop breaks. Nevertheless, as I've argued elsewhere, we need the continuum limit mathematics with its emergent divergences to explain why drops will (almost) always break with the same shape regardless of other details of how they were formed. $[5,8]$ Explanations do not necessarily have to be representative. And, in many (most) instances of explanations in applied mathematics, they are not.

\section{Conclusion}

The "problem of the applicability of mathematics in the natural sciences" has many facets. This paper has focused primarily on one - that of providing a viable account of how mathematical idealizations can play explanatory roles in physical theory. Most, if not all, attempts to answer this and related questions have their genesis in the debate about the ontological status of mathematical entities - the problem of the indispensability of mathematics. Perhaps, if one can show that mathematics plays an indispensable explanatory role in some physical explanations we may be able to infer to the existence of the abstract entities and properties referred to.

It is natural in this context to develop so-called mapping or structural accounts of the relationships between mathematical entities and their properties and the physical world. As Bueno and Colyvan say, "[m]athematics is a rich source of structures and when some mathematical theory finds applications in empirical science, it is clear that the mathematics captures certain important structural relations in the system in question." [10, p. 3]

I have argued, to the contrary, that it is not always clear that when a bit of mathematics gets applied in empirical science, it is because of some kind of static mirroring of empirical structure by the mathematics. My argument depends upon accepting the idea that idealizations can indeed be

\footnotetext{
${ }^{25}$ In [5] I do speak rather sloppily of genuine physical singularities. It is best to think instead in terms of some kind of genuine qualitative change in the system at a given scale.
} 
explanatory. (This is something both Pincock and Bueno and Colyvan accept.) But, it further depends upon recognizing that there are different types of explanatory idealizations. There are traditional or Galilean idealizations which are distinguished by the in principle ability to tell some kind of deidealizing story - one that demonstrates how ones model can be "improved" so as to provide a more accurate or more representative picture of the system modeled. There are also, I have argued, nontraditional idealizations in which the very idea of such a de-idealizing story is seriously problematic, if not incompatible with the explanatory features of the model. These nontraditional idealizations play essential explanatory roles involving operations or mathematical processes without representing the system(s) in question. And it is this fact that dooms mapping accounts to fail to provide a complete story about the explanatory applicability of mathematics.

I have suggested that a completely new approach is needed. It is an approach that looks to the world as the "driving influence" for how mathematics gets applied, rather than to fortuitous parallels or analogies between mathematical structures and physical structures. There is more to the applicability of mathematics than the fact that mathematics happens to be a very rich source of structures - a place to look for representations of physical structures. If this proposal is right, then there are strong reasons to deny Wigner's famous phrase concerning the "unreasonable effectiveness of mathematics in the natural sciences." Wigner said that the "appropriateness of the language of mathematics for the formulation of the laws of physics" is a "miracle" - "a wonderful gift which we neither understand nor deserve." [25, p. 237] If this proposal is right, then to the contrary, the world itself tells us that certain kinds of mathematical language is required for genuine understanding. 


\section{References}

[1] Alan Baker. Are there genuine mathematical explanations of physical phenomena? Mind, 114:223-238, 2005.

[2] Sorin Bangu. Thermodynamics taken seriously: Emergent phase transitions, data, and phenomena. forthcoming, 2008.

[3] Robert W. Batterman. Multiple realizability and universality. The British Journal for the Philosophy of Science, 51:115-145, 2000.

[4] Robert W. Batterman. The Devil in the Details: Asymptotic Reasoning in Explanation, Reduction, and Emergence. Oxford Studies in Philosophy of Science. Oxford University Press, 2002.

[5] Robert W. Batterman. Critical phenomena and breaking drops: Infinite idealizations in physics. Studies in History and Philosophy of Modern Physics, 36:225-244, 2005.

[6] Robert W. Batterman. Response to Belot's "Whose devil? Which details?". Philosophy of Science, 72:154-163, 2005.

[7] Robert W. Batterman. On the specialness of special functions (the nonrandom effusions of the divine mathematician). The British Journal for the Philosophy of Science, 58:235-262, 2007.

[8] Robert W. Batterman. Idealization and modelling. Synthese, Forthcoming.

[9] Gordon Belot. Whose devil? Which details? Philosophy of Science, 72:128-153, 2005.

[10] Otávio Bueno and Mark Colyvan. An inferential conception of the application of mathematics. Forthcoming.

[11] Craig Callender. Taking thermodynamics too seriously. Studies in History and Philosophy of Modern Physics, 32(4):539-533, 2001.

[12] Mark Colyvan. The Indispensability of Mathematics. Oxford University Press, Oxford, 2001.

[13] Mark Colyvan. Mathematics and aesthetic considerations in science. Mind, 111:69-74, 2002.

[14] Nicholaos J. Jones. Ineliminable Idealizations, Phase Transitions, and Irreversibility. PhD thesis, Ohio State University, Columbus, Ohio, 2006. 
[15] Leo P. Kadanoff. Statistical Physics: Statics, Dynamics, and Renormalization. World Scientific, Singapore, 2000.

[16] Mary Leng. Mathematical explanation. In Cellucci and Gillies, editors, Mathematical Reasoning, Heuristics and the Development of Mathematics, pages 167-189. King's College Publications, London.

[17] Ernan McMullin. Galilean idealization. Studies in History and Philosophy of Science, 16(3):247-273, 1985.

[18] Joseph Melia. Weaseling away the indispensability argument. Mind, 109:455-479, 2000.

[19] Joseph Melia. Response to Colyvan. Mind, 111:75-79, 2002.

[20] Margaret Morrison. Approximating the real: The role of idealization in physical theory. In Martin R. Jones and Nancy Cartwright, editors, Idealizations XII: Correctiong the Model; Idealization and Abstraction in the Sciences, pages 145-172. Rodopi, 2005.

[21] Ernest Nagel. The formation of modern conceptions of formal logic in the development of geometry. Osiris, 7:142-223, 1939.

[22] Christopher Pincock. A new perspective on the problem of applying mathematics. Philosophia Mathematica, 3(12):135-161, 2004.

[23] Christopher Pincock. Mathematical idealization. Philosophy of Science, 74:957-967, 2007.

[24] Christopher Pincock. A role for mathematics in the physical sciences. Nous, 41(2):253-275, 2007.

[25] Eugene P. Wigner. The unreasonable effectiveness of mathematics in the natural sciences. In Symmetries and Reflections: Scientific Essays of Eugene P. Wigner. Indiana University Press, 1967.

[26] Mark Wilson. Wandering Significance. Oxford University Press, 2006.

[27] Mark Wilson. Determinism and the mystery of the missing physics. PhilSci Archive, 2007.

[28] James Woodward. Making Things Happen: A Theory of Causal Explanation. Oxford Studies in Philosophy of Science. Oxford University Press, Oxford, 2003. 